\title{
Prevalencia de factores de riesgo que alteran la microbiota intestinal en niños escolares con sobrepeso y obesidad
}

\section{Prevalence of risk factors that alter the gut microbiota in overweight and obese schoolchildren}

DOI: $10.46932 / s f j d v 2 n 3-068$

Received in: May 1st, 2021

Accepted in: Jun 30th, 2021

\section{Ricardo Salas Flores \\ Endocrinólogo Pediatra., Master en Ciencias Médicas., Profesor Investigador.}

Current Institution: Coordinación de Educación en Salud e Investigación. Hospital General Regional No. 6, Madero, Tamaulipas, Instituto Mexicano del Seguro Social, México. Facultad de Medicina "Dr. Alberto Romo Caballero" Universidad Autónoma de Tamaulipas, Tampico, Tamaulipas, México.

Full address: Blvd. Adolfo López Mateos S/N, Col. Las Conchitas, Madero Tamaulipas.

\section{Brian González Pérez}

Médico Familiar., Master en Ciencias Médicas., Master en Innovación y Tecnología Educativa., Profesor Investigador.

Current Institution: Servicio de Atención Familiar. Unidad Médica Familiar 38, Tampico Tamaulipas, Instituto Mexicano del Seguro Social, México. Facultad de Medicina "Dr. Alberto Romo Caballero" Universidad Autónoma de Tamaulipas, Tampico, Tamaulipas, México.

Full address: Calle Argentina y Canadá 107 Col. Infonavit C.P. 89310, Tampico, Tamaulipas.

Email: brian.gonzalez.perez@gmail.com

\section{Gerardo Rivera Fernández}

Médico Pediatra.

Current Institution: Hospital General Regional No. 6, Madero, Tamaulipas, Instituto Mexicano del Seguro Social, México.

Full address: Blvd. Adolfo López Mateos S/N, Col. Las Conchitas, Madero Tamaulipas.

\section{Alejandra Salas Galarza \\ Médico Residente Endocrinologia.}

Current Institution: Centro Médico Nacional de Occidente, Guadalajara, Jalisco. Instituto Mexicano del Seguro Social, México.

Full address: Av. Belisario Domínguez 1000, Belisario Domínguez, CP 44329. Guadalajara, Jalisco.

\section{Liliana Aurora Carrillo Aguiar}

Médico Familiar., Master en Ciencias Médicas., Profesor Titular de la Residencia en Medicina Familiar. Current Institution: Unidad Médica Familiar 77, Madero Tamaulipas, Instituto Mexicano del Seguro Social, México.

Full address: Blvd. Adolfo López Mateos S/N, Col. Las Conchitas, Madero Tamaulipas.

\section{Verónica Olvera Mendoza}

Master en Finanzas., Profesor Investigador.

Current Institution: Facultad de Medicina "Dr. Alberto Romo Caballero" Universidad Autónoma de Tamaulipas, Tampico, Tamaulipas, México.

Full address: Centro Universitario Sur, Cto. Interior S/N CP 89337, Tampico, Tamaulipas 


\section{RESUMEN}

INTRODUCCION: Dentro de los factores de riesgo para presentar sobrepeso u obesidad, se reconoce al nacimiento por cesárea, a la ausencia de lactancia materna exclusiva y a la exposición a antimicrobianos a temprana edad como factores que favorecen al sobrepeso. Dentro del tema de la microbiota intestinal, los factores previamente mencionados para favorecer al sobrepeso, se encuentran también vinculados con la alteración de la microbiota intestinal, por lo que se sugiere que una microbiota intestinal alterada es un factor de riesgo para presentar sobrepeso y obesidad.

OBJETIVO: Demostrar la prevalencia de los factores de riesgo que alteran la microbiota intestinal en preescolares y escolares con sobrepeso u obesidad derechohabientes del Instituto Mexicano del Seguro Social en la región sur de Tamaulipas.

METODOS: Estudio observacional, descriptivo y transversal, donde se obtuvo a través de historias clínicas durante el periodo comprendido de marzo a diciembre 2020, en pacientes de ambos sexos, de los 2 a los 12 años de edad, los antecedentes de la vía de nacimiento, la alimentación de los primeros 6 meses de vida y la exposición a antibióticos durante el primer año de vida; fueron requeridos 385 pacientes para establecer un índice de confianza del 95\% y margen de error del 5\%, establecido por un cálculo para muestra de poblaciones finitas, conociendo que la prevalencia de sobrepeso y obesidad a nivel nacional es del 34\%. La recolección de datos se realizó en el Hospital General Regional \# 6 Cd. Madero Tamaulipas y en la Unidad Médico Familiar \#77 del IMSS, Cd. Madero, Tamaulipas. Se aplicaron estadísticas descriptivas y medidas de tendencia central.

RESULTADOS: De los 385 pacientes con sobrepeso u obesidad estudiados, el $60 \%$ fueron obtenidos por cesárea, el $74.5 \%$ recibió alimentación entre fórmula exclusiva y combinada y el $42 \%$ recibieron antibióticos en el primer año de vida.

CONCLUSIONES: La prevalencia de cesáreas según la OMS en México en el 2017 es del 45\%, en este estudio se encontró una prevalencia del $60 \%$, la alimentación por leche materna exclusiva en este país tiene una prevalencia del $15.5 \%$ y en este estudio fue de $25.5 \%$; no se cuenta con un estudio de prevalencia de uso de antibióticos en el primer año de vida para poder compararse con el $42 \%$ registrado en este estudio.

Palabras Clave: microbiota intestinal, sobrepeso, obesidad, cesáreas, lactancia materna, prebióticos, probióticos, antimicrobianos.

\footnotetext{
ABSTRACT

INTRODUCTION: Among the risk factors for being overweight or obese, birth by cesarean section, the absence of exclusive breastfeeding and exposure to antimicrobials at an early age are recognized as factors that favor overweight. Within the subject of the intestinal microbiota, the previously mentioned factors to favor overweight are also linked to the alteration of the intestinal microbiota, which is why it is suggested that an altered intestinal microbiota is a risk factor for being overweight and obese.

OBJECTIVE: To demonstrate the prevalence of risk factors that alter the intestinal microbiota in preschool children and schoolchildren with overweight or obesity.

METHODS: Observational, descriptive and cross-sectional study, where it was obtained through medical records in patients from 2 to 12 years of age, the history of the route of birth, the diet of the first 6 months of life and the exposure to antibiotics during The first year of life; 385 patients were required to establish a confidence index of $95 \%$ and a margin of error of 5\%, established by a calculation for a sample of finite populations, knowing that the prevalence of overweight and obesity at the national level is $34 \%$ and that the population age included in this study is 22 million in the country. Data collection was carried out at Hospital General Regional \# 6 Cd. Madero Tamaulipas and at Family Medical Unit \# 77 of the IMSS, Cd. Madero, Tamaulipas. Descriptive statistics and measures of central tendency were applied.
} 
RESULTS: Of the 385 overweight or obese patients studied, $60 \%$ were obtained by cesarean section, $74.5 \%$ received feeding between exclusive and combined formula, and $42 \%$ received antibiotics in the first year of life.

CONCLUSIONS: The prevalence of caesarean sections according to the WHO in Mexico in 2017 is $45 \%$, in this study a prevalence of $60 \%$ was found, exclusive breast milk feeding in this country has a prevalence of $15.5 \%$ and in this study it was $25.5 \%$; There is no study on the prevalence of antibiotic use in the first year of life to be able to compare it with the $42 \%$ registered in this study.

Keywords: intestinal microbiota, overweight, obesity, caesarean sections, breastfeeding, prebiotics, probiotics, antimicrobials.

\section{INTRODUCCION}

La microbiota intestinal es una comunidad de microorganismos vivos residentes en el tubo digestivo indispensable para el correcto crecimiento corporal, el desarrollo de la inmunidad y la nutrición; al conjunto formado por microorganismos, sus genes y sus metabolitos se le denominan microbioma (1). La forma de nacimiento, el medio ambiente temprano y la lactancia materna son determinantes clave para el desarrollo de una microbiota intestinal sana; una microbiota aberrante, puede ser un factor detrás del aumento progresivo de enfermedades no transmisibles como alergias, autoinmunidades y obesidad (2).

Al nacimiento, la superficie de las mucosas del neonato se exponen al microbioma materno $(3,4)$, Hay una colonización bacteriana masiva del neonato ante la exposición con la flora vaginal, fecal y de piel materna (5); el intestino de los recién nacidos obtenidos vía vaginal es colonizado por el microbioma rectal y vaginal materno, representado por una comunidad predominante de especies de Lactobacillus y Prevotella (3), especies anaerobias facultativas como Escherichia coli, especies de Staphylococcus y de Streptococcus colonizan al intestino y producen un ambiente anaerobio que permite el crecimiento de anaerobios estrictos como especies de Bacteroides y de Bifidobacterium (6); los Lactobacillus ayudan a mantener un $\mathrm{pH}$ vaginal bajo limitando la sobreproducción bacteriana y su ascenso hacia el útero, donde podrían infectar al líquido amniótico, placenta y feto. Se demostró aumento de especies de Lactobacillus y Bifidobacterium en mujeres embarazadas de 35 a 37 semanas de gestación, tanto en microbiota vaginal como rectal (7). La distribución de la microbiota femenina cambia en relación a mujeres embarazadas y no embarazadas, demostrando un aumento en la abundancia de especies de Lactobacillus en mujeres embarazadas, así como la composición de la microbiota menos diversa conforme se acerca a periodo de término (8). Las comunidades microbianas tempranas se expanden rápidamente y se diversifican en las primeras semanas de vida (3).

Los más grandes modificadores negativos de la microbiota intestinal son tres componentes: Alimentación a través de fórmula láctea, nacimiento por cesárea y uso de antibióticos a temprana edad 
(menores de un año de edad) (7). Un factor muy importante para el moldeamiento de la microbiota intestinal es la leche materna, la academia americana de pediatría hace fuerte recomendación de alimentación por leche materna en la mayoría de las circunstancias, la leche materna contiene anticuerpos que contribuyen a la inmunidad del huésped, demostrando disminución de riesgo para contraer enfermedades contagiosas, a grandes rasgos disminuye mortalidad neonatal (9).

La leche materna contiene ácidos grasos, vitaminas, minerales, inmunoglobulina A (IgA) y oligosacáridos. El "factor bífido", descubierto en 1926 por Schonfeld, se encontró que promueve el crecimiento de una flora comensal conocida como Bifidobacterium bifidus; actualmente se reconoce en los oligosacáridos de la leche materna este factor bífido (9).

Agregado a los componentes de la leche materna, ésta también contiene bacterias que comúnmente se encuentran en piel y en sistemas entéricos, como Streptococcus, Staphylococcus, Escherichias, Enterococcus, Serratia, Corynebacterium, Pseudomonas, Ralstonia, Propionibacterium, Sphingomonas, Bradyrhizobiaceae, así como también especies de Bifidobacterium y Lactobacillus (10). El microbioma de la leche materna es variado por la edad gestacional en la que se obtuvo el producto, mostrando mayor concentración de especies de Bifidobacterium en la leche materna de madres que llevaron su embarazo al término a comparación de leche materna de madres que terminaron su embarazo en etapas tempranas (11), también diferenciado el microbioma por la etapa de producción de leche en la que se encuentra, iniciando con leche calostro que es la de mayor diversidad bacteriana y disminuyendo dicha diversidad conforme madura la leche (12); se ha documentado aumento de cantidad de Staphylococcus con menor cantidad de Bifidobacterium en leche materna de madres obesas. Por si sola la leche materna contiene su propio microbioma único que contribuye a la formación de comunidades bacterianas el intestino neonatal, modulando la microbiota (9).

La leche materna es única por su alta concentración de oligosacáridos en comparación con la leche de otros mamíferos. Los oligosacáridos de la leche materna funcionan como prebióticos $(10,13)$, son una fuente importante de carbono para la fermentación de la microbiota, moldeando su composición durante el primer mes de vida hacia un ecosistema predominantemente dominado por especies de Bifidobacterium y Lactobacillus, entre estas se promueve en su mayoría el crecimiento de Bifidobacterium infantis, y sirve como organismo comensal que previene crecimiento de bacterias patogénicas en el intestino neonatal, promueven funcionamiento de la barrera intestinal, previene adhesión vírica, bacteriana y de protozoarios, funcionan como receptor para patógenos, reduciendo la incidencia de infecciones neonatales (14); incluso se vincula la actividad de los oligosacáridos de leche materna como razón por la que la incidencia de infección vertical de VIH es menor al $10 \%$ en los neonatos que la adquieren a través de leche materna (8). 
El efecto protector de la lactancia no sólo está confinado a una menor morbilidad y mortalidad a corto plazo, pues éste se extiende a etapas posteriores y hay clara evidencia para enfermedades crónicas como dermatitis, asma, exceso de peso, diabetes tipos 1 y 2 y leucemia (15). La sinergia de los componentes prebióticos y probióticos de la leche materna provee al lactante de una microbiota intestinal más estable y uniforme a comparar con los lactantes alimentados de fórmula láctea (16).

La introducción de fórmulas lácteas o alimentos sólidos en periodo postnatal perturba la colonización y proliferación de la microbiota intestinal neonatal y reduce los beneficios de la alimentación exclusiva de leche materna, por consiguiente aumenta la prevalencia de Clostridium difficile, Bacteroides fragilis y E. coli $(17,18)$. La alimentación con fórmula, incluso en pequeñas cantidades durante la alimentación por leche materna puede alterar la estructura de la microbiota por disminución de la concentración de su efecto prebiótico y probiótico, promoviendo el crecimiento de agentes diferentes a especies de Bifidobacterium (19), alterando el desarrollo apropiado del sistema inmunológico y posteriormente altera al metabolismo, contribuyendo a la predisposición hacia la obesidad (7).

El patrón de crecimiento de los niños alimentados con leche humana es diferente a los alimentados con fórmula láctea, y éste, junto con la composición de la leche humana ha sugerido a la lactancia materna como posible mecanismo protector contra el sobrepeso, la obesidad, al igual que otras enfermedades crónicas relacionadas con la nutrición, como leucemia o diabetes (15); los lactantes alimentados exclusivamente al seno materno mayores de 12 meses de edad, muestran menor riesgo de sobrepeso u obesidad, explicado por las hormonas de la leche materna, entre estas las más estudiadas son leptina e insulina, que inducen efectos a largo plazo en la regulación del peso y la sensibilidad a la insulina del lactante (20). México experimenta un fenómeno de abandono de la práctica de lactancia materna exclusiva (de $22.3 \%$ en 2006 a $14.4 \%$ en 2012). Estudios nacionales señalan que las barreras para la lactancia materna incluyen desde aquellas de índole individual hasta las relacionadas con el ambiente sociocultural y de políticas en el país (15).

El factor económico de la lactancia materna representa ahorros en la familia asociados con los gastos de fórmulas lácteas, consultas médicas y medicamentos. Las recientes estimaciones para México indican que el costo anual de prácticas inadecuadas de la lactancia materna es entre \$11 100 y \$36 200 millones por año, donde un porcentaje considerable de este gasto se atribuye a la compra de fórmulas lácteas (11 a 38\%). Dado que la lactancia materna exclusiva menor a 6 meses ha descendido mucho más en la población de escasos recursos (medio rural y zona sur del país) estos gastos recaen cada vez más en las familias más vulnerables (15). 
En el caso de las cesáreas, esta no transmite la variedad de microorganismos que se transmiten a través del parto (especies de Lactobacillus, Prevotella y Fusobacterium) (21), la cesárea transmite especies de Staphylococcus, Propionobacterium y Corynebacterium que se encuentran en la piel. Al ser colonizado el epitelio intestinal neonatal por bacterias de la piel, estas favorecen la colonización por Clostridium y por consiguiente no permite la colonización oportuna de especies de Bacteroides y de Bifidobacterium (22); se han documentado diferentes especies en la microbiota intestinal en pacientes obtenidos por cesárea a diferencia de los que fueron obtenidos por parto, aún bajo seguimiento al año, a los dos años e incluso estudiado hasta los 7 años de vida $(23,24)$. El nacimiento por cesárea de manera aislada(como único factor para alteración de microbiota intestinal neonatal) se documenta aumento de riesgo de enfermedad celiaca, asma, alergias, diabetes tipo 1, tipo 2, obesidad, siendo esta ultima el $46 \%$ $(7,9)$.

Según la Organización Mundial de la Salud (OMS) desde 1985, la cesárea es indispensable en el 10 a 15\% de los partos; la tasa de partos por cesárea ha aumentado consistentemente en los últimos 50 años, siendo de aproximadamente $5 \%$ en los años 60, ascendiendo hasta $35 \%$ al año 2000, posteriormente $49 \%$ en 2010 , manteniendo su pico máximo hasta 2014 , con un descenso al $45 \%$ en 2015, la cesárea es la cirugía más realizada en los hospitales de segundo y tercer nivel $(25,26)$.

Por último, los antibióticos, estos alteran al microbioma intestinal, independiente de la etapa en la que se encuentra(prenatal, durante el nacimiento y postnatal), se ha documentado desde el momento de su nacimiento, en quienes se realizó profilaxis antimicrobiana preparto o previa a la cesárea, obteniendo como muestra la primer evacuación del recién nacido, mostrando una menor diversidad bacteriana, así como disminución de la abundancia de especies de Lactobacillus y Bifidobacterium, con asociaciones similares secundarias a la administración de antibióticos postnatales $(27,28)$. El uso de antibióticos durante el embarazo aumenta riesgo de obesidad infantil y asma, niños expuestos a antibióticos prenatales en segundo o tercer trimestre mostraron $84 \%$ más riesgo de obesidad a comparar con niños no expuestos (29). Actualmente, hasta el 50\% de todos los antibióticos prescritos a personas no son necesarios o no son óptimamente eficaces según lo prescrito (30).

La prescripción inadecuada de antibióticos en relación con infecciones respiratorias agudas (IRAS) e infecciones gastrointestinales/diarreicas agudas (EDAS), en el sector público, es el tema más ampliamente documentado sobre uso de medicamentos en México, entre 1980 y 1990, el 60 a $80 \%$ de los pacientes con IRAS y EDAS recibían antibióticos cuando en realidad su uso se justificaba en solo 10 a $15 \%$ de los casos; entre 70 y $80 \%$ de recomendaciones terapéuticas proporcionadas por empleados de farmacias para IRAS, EDAS e infecciones urinarias, mayoritariamente antibióticos, son incorrectas (31). 
Con información de las Encuestas Nacionales de Nutrición en México, desde el año 1988 se ha podido documentar la tendencia permanente al aumento del sobrepeso y la obesidad en escolares y adolescentes, Para el año 2016, niñas preescolares presentan 5.8\% de sobrepeso y obesidad, mientras los niños un $6.5 \%$, en escolares las niñas con una prevalencia del $32 \%$ y niños $33.7 \%$. En mujeres adolescentes hubo in incremento de sobrepeso y obesidad del 2012 a 2016 de $35.8 \%$ a 39.2\%, mientras que hombres bajó de $34.1 \%$ a $33.5 \%$ respectivamente (32).

Durante los primeros años de vida y antes de los 18 años de edad, la obesidad tiene consecuencias importantes para la salud física y mental. Las niñas y niños obesos tienen mayor probabilidad de padecer enfermedades como síndrome metabólico, diabetes, hipertensión, enfermedades del corazón y problemas ortopédicos, entre otros, en el futuro. Asimismo, existe una fuerte asociación de este padecimiento a edades tempranas con problemas de autoestima y discriminación (32).

De los factores previamente mencionados para favorecer al sobrepeso, se encuentran también vinculados con la alteración de la microbiota intestinal, por lo que se sugiere que una microbiota intestinal alterada es un factor de riesgo para presentar sobrepeso y obesidad. El objetivo del presente estudio fue demostrar la prevalencia de los factores de riesgo que alteran la microbiota intestinal en preescolares y escolares con sobrepeso u obesidad.

\section{METODOS}

Estudio observacional, descriptivo y transversal, donde se obtuvo a través de historias clínicas durante el periodo comprendido de marzo a diciembre 2020, en pacientes de ambos sexos, de los 2 a los 12 años de edad, los antecedentes de la vía de nacimiento, la alimentación de los primeros 6 meses de vida y la exposición a antibióticos durante el primer año de vida; fueron requeridos 385 pacientes para establecer un índice de confianza del 95\% y margen de error del 5\%, establecido por un cálculo para muestra de poblaciones finitas, conociendo que la prevalencia de sobrepeso y obesidad a nivel nacional es del 34\%. La recolección de datos se realizó en el Hospital General Regional \# 6 Cd. Madero Tamaulipas y en la Unidad Médico Familiar \#77 del IMSS, Cd. Madero, Tamaulipas. El protocolo de investigación fue aprobado por el Comité Local de Investigación en Salud 2801 del Instituto Mexicano del Seguro Social. El análisis de los resultados fue descriptivo, de prevalencia; se obtuvo la frecuencia en la que se presentaba cada uno de los factores de riesgo mencionados, así como la frecuencia de más de un factor de riesgo por sujeto y la frecuencia de los que no presentaron ninguno de los factores de riesgo. Se determinó la prevalencia de cesáreas, de alimentación con fórmulas o lactancia materna no exclusiva y la prevalencia del uso de algún antimicrobiano en el primer año de vida en un periodo mayor a 5 días, la prevalencia combinada de dos factores de riesgo, la prevalencia de los tres factores de riesgo 
estudiados, así como la prevalencia de los sujetos que quedaron libres de factores de riesgo. Una vez obtenida la prevalencia de cada factor, se obtuvo también la prevalencia de obesidad en quienes cumplieron con los tres factores de riesgo positivos y quienes no tuvieron ningún factor de riesgo presente.

\section{RESULTADOS}

Se estudiaron un total de 385 pacientes, en los que se encontraron 208 niños (54\%) y 177 niñas (46\%). La frecuencia de distribución por etapas de edad fue representada en la tabla 1.

\begin{tabular}{|l|l|l|}
\hline Tabla 1. Distribución de etapas. & \multicolumn{2}{|l|}{} \\
\hline Rango de edad & Frecuencia & Porcentaje \\
\hline Preescolar (2-6 años) & 186 & $48.3 \%$ \\
\hline Escolar (7-12 años) & 199 & $51.7 \%$ \\
\hline
\end{tabular}

Respecto a la prevalencia de cada factor de riesgo, se obtuvo la prevalencia de cada factor de riesgo, representada en la tabla 2 .

\begin{tabular}{|l|l|l|}
\hline Tabla 2. Prevalencia por cada factor de riesgo \\
\hline Variable & Frecuencia (total 385) & Porcentaje \\
\hline Nacimiento por cesárea & 233 & $60.52 \%$ \\
\hline Antibióticos 1er año de vida & 162 & $42.08 \%$ \\
\hline Lactancia no exclusiva & 287 & $74.54 \%$ \\
\hline
\end{tabular}

De los 385 pacientes con sobrepeso u obesidad estudiados, 35 pacientes no tuvieron factores de riesgo alguno, mientras que 87 pacientes presentaron los tres factores de riesgo al mismo tiempo, representados en la tabla 3 .

Tabla 3. Presencia de los tres factores de riesgo (Cesárea, Lactancia no exclusiva y antibióticos)

\begin{tabular}{|l|l|l|}
\hline Factores de riesgo & Frecuencia & Porcentaje \\
\hline Presentes & 87 & $22.6 \%$ \\
\hline Ausentes & 35 & $9.09 \%$ \\
\hline
\end{tabular}

A los pacientes que se les detectó la presencia o ausencia de los tres factores de riesgo estudiados, se determinó la prevalencia de obesidad (percentil mayor a 97) entre ellos, es representada en la tabla 4. 


\section{Tabla 4. Prevalencia de obesidad}

\begin{tabular}{|l|l|l|}
\hline Factores de riesgo & Frecuencia & Porcentaje \\
\hline Presentes & 41 & $47.12 \%$ \\
\hline Ausentes & 7 & $20 \%$ \\
\hline
\end{tabular}

La prevalencia de positividad en por lo menos uno de los tres factores de riesgo estudiados fue del $90.91 \%$

\section{DISCUSIÓN}

Durante este estudio descriptivo, se encontró una prevalencia elevada de los factores de riesgo que alteran la microbiota intestinal en el universo estudiado, que fue el de los pacientes pediátricos de edad comprendida de 2 a 12 años y que contaban ya con un diagnóstico de sobrepeso u obesidad. Se comprobó la hipótesis al demostrar que el $90.9 \%$ de la población estudiada cuenta con por lo menos un factor de riesgo para alteración de la microbiota intestinal.

Se cumplió con el objetivo general de encontrar la prevalencia de los factores de riesgo para alteración de la microbiota intestinal, y uno de los objetivos generales fue el demostrar cual es el factor de riesgo que tiene mayor prevalencia entre los estudiados, que fue el de la lactancia materna no exclusiva, siendo un $74.54 \%$ su prevalencia, sin embargo, por estadística nacional la lactancia no exclusiva es menor al $15 \%$ en nuestro país de acuerdo a registros del 2012, faltaría un registro actualizado para demostrar si ha aumentado la lactancia materna exclusiva y poder compararla con la de este estudio; por el momento da a entender este resultado que, aunque la lactancia materna exclusiva es pobre en el país, en este estudio se encontró mayor prevalencia de lactancia materna exclusiva que con el resto de la población.

Ante lo mencionado de la lactancia materna, el factor de riesgo que si fue mayor al de los registros nacionales fue el de los nacimientos por cesárea, siendo $60 \%$ en este estudio y $45 \%$ en la población general. No se puede demostrar por medio de este estudio cuál de los tres factores de riesgo tiene más impacto en la alteración de la microbiota intestinal. No se puede realizar una comparación de la prevalencia de consumo de antibióticos en el primer año de vida porque no contamos con dicha información en estudios publicados recientemente.

\section{CONCLUSIONES.}

El sobrepeso y obesidad están vinculados a los factores de riesgo que alteran la microbiota intestinal. 
En una población infantil que padece de sobrepeso y obesidad, más del 90\% tienen algún factor de riesgo positivo para alteración de la microbiota intestinal.

Es una situación de urgencia disminuir la indicación de cesáreas injustificadas, fomentar la lactancia materna exclusiva totalmente y reconsiderar la clínica del paciente antes de dar tratamientos antimicrobianos. 


\section{REFERENCIAS BIBLIOGRÁFICAS}

1. Icaza-Chávez, Microbiota intestinal en la salud y la enfermedad, Revista de Gastroenterología de México 2013; 78 (4): 240-248 https://doi.org/10.1016/j.rgmx.2013.04.004

2. Miettinen R, Hermansson H, Merikukka M, Gissler M, Isolauri E. Mode of delivery--impact on risk of noncommunicable diseases. J Allergy Clin Immunol. 2015;136(5):1398-1399. https://doi:10.1016/j.jaci.2015.05.032

3. Perdijk O, Marsland BJ. The microbiome: toward preventing allergies and asthma by nutritional intervention. Curr Opin Immunol. 2019; 60: 10-18. https://doi:10.1016/j.coi.2019.04.001

4. Biasucci G, Rubini M, Riboni S, Morelli L, Bessi E, Retetangos C. Mode of delivery affects the bacterial community in the newborn gut. Early Hum Dev. 2010;86 Suppl 1:13-15. https://doi:10.1016/j.earlhumdev.2010.01.004

5. Palmer C, Bik EM, DiGiulio DB, Relman DA, Brown PO. Development of the human infant intestinal microbiota. PLoS Biol. 2007;5(7):e177. https://doi:10.1371/journal.pbio.0050177

6. Pantoja-Feliciano IG, Clemente JC, Costello EK, et al. Biphasic assembly of the murine intestinal microbiota during early development. ISME J. 2013;7(6):1112-1115. https://doi:10.1038/ismej.2013.15

7. Mueller NT, Bakacs E, Combellick J, Grigoryan Z, Dominguez-Bello MG. The infant microbiome development: mom matters. Trends Mol Med. 2015;21(2):109-117. https://doi:10.1016/j.molmed.2014.12.002

8. Aagaard K, Riehle K, Ma J, et al. A metagenomic approach to characterization of the vaginal microbiome signature in pregnancy. PLoS One. 2012;7(6):e36466. https://doi:10.1371/journal.pone.0036466

9. Valentine G, Prince A, Aagaard KM. The Neonatal Microbiome and Metagenomics: What Do We Know and What Is the Future?. Neoreviews. 2019;20(5):e258-e271. https://doi:10.1542/neo.20-5e258

10. Hunt KM, Foster JA, Forney LJ, et al. Characterization of the diversity and temporal stability of bacterial communities in human milk. PLoS One. 2011;6(6):e21313. https://doi:10.1371/journal.pone.0021313

11. Khodayar-Pardo P, Mira-Pascual L, Collado MC, Martínez-Costa C. Impact of lactation stage, gestational age and mode of delivery on breast milk microbiota. J Perinatol. 2014;34(8):599-605. https://doi:10.1038/jp.2014.47.

12. Cabrera-Rubio R, Collado MC, Laitinen K, Salminen S, Isolauri E, Mira A. The human milk microbiome changes over lactation and is shaped by maternal weight and mode of delivery. Am J Clin Nutr. 2012;96(3):544-551. https://doi:10.3945/ajcn.112.037382

13. Coppa GV, Bruni S, Morelli L, Soldi S, Gabrielli O. The first prebiotics in humans: human milk $\begin{array}{lllll}\text { oligosaccharides. } & \mathrm{J} & \text { Clin } & \text { Gastroenterol. } & \text { 2004;38(6 }\end{array}$ https://doi:10.1097/01.mcg.0000128926.14285.25 
14. Sudo N, Sawamura S, Tanaka K, Aiba Y, Kubo C, Koga Y. The requirement of intestinal bacterial flora for the development of an $\mathrm{IgE}$ production system fully susceptible to oral tolerance induction. J Immunol. 1997;159(4):1739-1745.

15. González, Teresita, et al. Lactancia Materna en México, CONACYT (Consejo Nacional de Ciencia y Tecnología) vol 1. 2016; pp 178.

16. Bode L. Human milk oligosaccharides: prebiotics and beyond. Nutr Rev. 2009;67 Suppl 2:S183S191. https://doi:10.1111/j.1753-4887.2009.00239.x

17. Penders J, Vink C, Driessen C, London N, Thijs C, Stobberingh EE. Quantification of Bifidobacterium spp., Escherichia coli and Clostridium difficile in faecal samples of breast-fed and formula-fed infants by real-time PCR. FEMS Microbiol Lett. 2005;243(1):141-147. https://doi:10.1016/j.femsle.2004.11.052

18. Fallani M, Young D, Scott J, et al. Intestinal microbiota of 6-week-old infants across Europe: geographic influence beyond delivery mode, breast-feeding, and antibiotics. J Pediatr Gastroenterol Nutr. 2010;51(1):77-84. https://doi:10.1097/MPG.0b013e3181d1b11e

19. Guaraldi F, Salvatori G. Effect of breast and formula feeding on gut microbiota shaping in newborns. Front Cell Infect Microbiol. 2012;2:94. Published 2012 Oct 16. https://doi:10.3389/fcimb.2012.00094

20. Demmelmair H, Koletzko B. Variation of Metabolite and Hormone Contents in Human Milk. Clin Perinatol. 2017;44(1):151-164. https://doi:10.1016/j.clp.2016.11.007

21. Dominguez-Bello MG, Costello EK, Contreras M, et al. Delivery mode shapes the acquisition and structure of the initial microbiota across multiple body habitats in newborns. Proc Natl Acad Sci U S A. 2010; 107(26): 11971-11975. https://doi:10.1073/pnas.1002601107

22. Jakobsson HE, Abrahamsson TR, Jenmalm MC, et al. Decreased gut microbiota diversity, delayed Bacteroidetes colonisation and reduced Th1 responses in infants delivered by caesarean section. Gut. 2014;63(4):559-566. https://doi:10.1136/gutjnl-2012-303249

23. Huurre A, Kalliomäki M, Rautava S, Rinne M, Salminen S, Isolauri E. Mode of delivery effects on gut microbiota and humoral immunity. Neonatology. 2008;93(4):236-240. https://doi:10.1159/000111102

24. Salminen S, Gibson GR, McCartney AL, Isolauri E. Influence of mode of delivery on gut microbiota composition in seven year old children. Gut. 2004;53(9):1388-1389. https://doi:10.1136/gut.2004.041640

25. Secretaría de Salud. Informe Sobre la Salud de los Mexicanos, Diagnóstico general del sistema nacional de salud. Primera edición. 2016, pp 152

26. Bernal-García, Celina et al. Cesárea: situación actual y factores asociados en México, vol 11. 2018; pp. 6. 
27. Jauréguy F, Carton M, Panel P, Foucaud P, Butel MJ, Doucet-Populaire F. Effects of intrapartum penicillin prophylaxis on intestinal bacterial colonization in infants. $\mathrm{J}$ Clin Microbiol. 2004;42(11):5184-5188. https://doi:10.1128/JCM.42.11.5184-5188.2004

28. Tanaka S, Kobayashi T, Songjinda P, et al. Influence of antibiotic exposure in the early postnatal period on the development of intestinal microbiota. FEMS Immunol Med Microbiol. 2009;56(1):80-87. https://doi:10.1111/j.1574-695X.2009.00553.x

29. Mueller NT, Whyatt R, Hoepner L, et al. Prenatal exposure to antibiotics, cesarean section and risk of childhood obesity. Int J Obes (Lond). 2015;39(4):665-670. https://doi:10.1038/ijo.2014.180

30. Diario Oficial de la Federación, Acuerdo por el que se declara la obligatoriedad de la Estrategia Nacional de Acción contra la Resistencia a los Antimicrobianos, 2018. http://www.dof.gob.mx/nota_detalle.php?codigo=5525043\&fecha=05/06/2018

31. Dreser, A, et al. Uso de antibióticos en México: revisión de problemas y políticas, Salud Pública de México, Vol.50, 2018; pp. 8.

32. Shamah-Levy, Teresa, et al. Sobrepeso y obesidad en niños y adolescentes en México, actualización de la Encuesta Nacional de Salud y Nutrición de Medio Camino. Vol 60. 2016; pp. 10. 